\title{
ANAESTHESIA FOR LAPAROSCOPIC EXCISION OF PHEOCHROMOCYTOMA AND PARAGANGLIOMAS IN PAEDIATRIC POPULATION- OUR INSTITUTIONAL EXPERIENCE
}

\author{
Kunigal Ravishankar Chandrakala1, Dammaningala Venkataramaiah Bhagya2, Yabagodu Ramayya Chandrika ${ }^{3}$
}

${ }^{1}$ Associate Professor, Department of Paediatric Anaesthesia, Indira Gandhi Institute of Child Health, Bengaluru, Karnataka.

${ }^{2}$ Assistant Professor, Department of Paediatric Anaesthesia, Indira Gandhi Institute of Child Health, Bengaluru, Karnataka.

3 Professor and HOD, Department of Paediatric Anaesthesia, Indira Gandhi Institute of Child Health, Bengaluru, Karnataka.

\section{BACKGROUND}

ABSTRACT

Pheochromocytoma presents a challenge to the anaesthesiologists because of its clinical features and implications, especially in paediatric population. The most crucial moments are induction and haemodynamic oscillations.

Our review aims at discussion of pharmacological strategies regarding perioperative management of paediatric pheochromocytoma in our institution.

The objective of this study is to look for various strategies aiding in better perioperative anaesthetic care of pheochromocytoma patients in future.

\section{MATERIALS AND METHODS}

We managed 7 children aged between 8 and 14 years with pheochromocytoma over a period of 9 years from 2006 to 2015 . Hypertension, headache and sweating were the most common clinical features noted. Early involvement of anaesthesiologist in preoperative management was achieved with phenoxybenzamine in all the patients. Main anaesthetic drugs used were thiopental sodium, morphine, atracurium, dexmedetomidine and magnesium sulphate.

\section{RESULTS}

There was successful perioperative management of all children. Two of the seven patients underwent intraoperative conversion of surgery to open laparotomy, two had severe post-operative hypotension which was well managed on time and recurrence of pheochromocytoma was seen in one patient. All children were normotensive when discharged. There were no deaths reported on long-term followup.

\section{CONCLUSION}

Adequate preoperative preparation, modern anaesthetic drugs, good collaboration with surgeons and proper post-operative care can improve the outcome and complications.

\section{KEYWORDS}

PCC- Pheochromocytoma, HTN- Hypertension, PGL- Paragangliomas.

HOW TO CITE THIS ARTICLE: Chandrakala KR, Bhagya DV, Chandrika YR. Anaesthesia for laparoscopic excision of pheochromocytoma and paragangliomas in paediatric population- our institutional experience. J. Evolution Med. Dent. Sci. 2017;6(93):6770-6776, DOI: 10.14260/jemds/2017/1466

\section{BACKGROUND}

Pheochromocytoma (PCC) is a rare neuroendocrine tumour in children, commonly seen in preadolescent boys with great tendency for familial occurrence and higher bilateral incidents.[1] The name 'Pheochromocytoma' was coined by Pick in 1912, then it was von Frankel who recognised and studied extensively in later years.[2,3] The tumour produces excessive amount of catecholamines causing dramatic lifethreatening cardiovascular effects. Current knowledge of the pathophysiology, advances in adrenergic pharmacology and newer drugs have made the management easy, thus reducing the perioperative morbidity and mortality rate significantly compared to earlier times.[2]

'Financial or Other Competing Interest': None.

Submission 27-10-2017, Peer Review 21-11-2017,

Acceptance 27-11-2017, Published 11-12-2017.

Corresponding Author:

Dammaningala Venkataramaiah Bhagya,

\#49, $3^{\text {rd }}$ Block,

$3^{\text {rd }}$ Stage, Basaveshwaranagar,

Bengaluru-560079,

Karnataka.

E-mail: dvanitha207@gmail.com

DOI: $10.14260 /$ jemds $/ 2017 / 1466$

(c) $(7)$
In the fifth week of foetal development, neuroblastic cells migrate from the thoracic neural crest to form the sympathetic chains and preaortic ganglia. These cells are the precursors of neuroblastomas and ganglioneuromas.[4] Chromaffin cells migrate second time to the adrenal medulla and settle near the sympathetic ganglia, vagus nerve, paraganglia and carotid arteries. In children, pheochromocytoma is more frequently associated with other familial syndromes such as neurofibromatosis, von HippelLindau disease, tuberous sclerosis, Sturge-Weber syndrome and also as a component of multiple endocrine neoplasia (MEN) syndromes.

Pheochromocytomas are catecholamine secreting tumours in the adrenal medulla, whereas paragangliomas are located anywhere in the body from skull to pelvis but commonly arise in head and neck, in the abdomen near the renal vessels or the organ of Zuckerkandl, which is the largest extra-adrenal collection of chromaffin tissue. The term "pheo" is often used interchangeably with Paraganglioma (PGL), but there is a distinction between these two types due to underlying differences in genetics, clinical presentation and malignant potential.[3]

The term paraganglioma refers to any extra-adrenal or non-functional tumour of paraganglion system, whereas 
functional tumour is referred as extra-adrenal pheochromocytomas.[4] Malignant pheochromocytomas are rare in paediatric population, usually they are locally invasive, may spread to distant areas that do not contain chromaffin cells like liver, lung, bone and lymph nodes.[5] Features of malignant tumours include extra-adrenal location, tumour necrosis, absence of hyaline globules, coarse nodularity of the tumour, high proliferative index size more than $5 \mathrm{cms}$. The mean survival rate in patients with malignant pheochromocytomas is $40 \%{ }^{[3]}$

\section{Genetic Basis of Pheochromocytoma}

It may be inherited as an autosomal dominant trait. Genes like SDHD, SDHB and SDHC that belongs to the mitochondrial complex II have been involved in non-syndromic pheochromocytomas and paragangliomas. $[4,6]$

\section{Catecholamine Biosynthesis and Metabolism}

The important steps in the biosynthesis and metabolism of catecholamines-

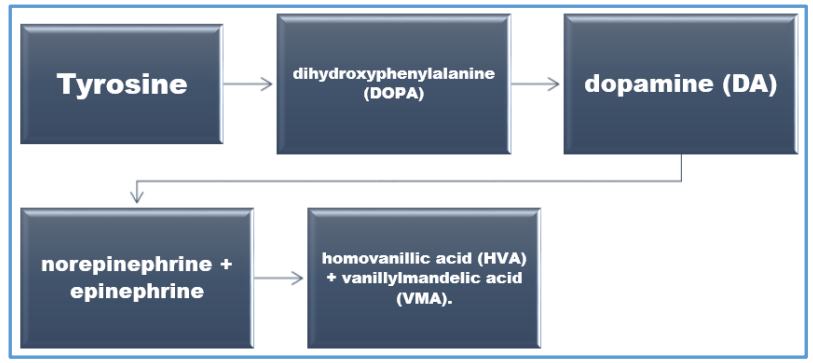

Figure 1. Biosynthesis and Metabolism of Catecholamines

The biosynthesis and storage of catecholamine in chromaffin cell tumours may differ from normal medulla, yet the granules are morphologically and functionally similar. The increase in tissue turnover suggests an alteration in the regulation of the catecholamine biosynthesis and feedback inhibition of tyrosine hydroxylase and key enzyme in the production of catecholamines. In the sympathetic system the reaction closes at noradrenaline, but in the adrenal gland and chromaffin cells methylation to adrenaline occurs. If there is little to no elevations of adrenaline excretion in the urine in a patient with pheochromocytoma, the tumour is probably located in sympathetic chain and not in the adrenal gland.

Three major metabolic pathways for adrenaline and noradrenaline are deamination, 0-methylation and conjugation.

\section{Catecholamines in Pheochromocytomas}

PCCs, unlike the normal adrenal medulla are not innervated, hence catecholamine release is not initiated by neural impulses. Changes in direct flow, pressure, chemicals, drugs and angiotensin II may initiate the release of catecholamines into the circulation. Most PCCs in children predominantly produce norepinephrine unlike the normal adrenal medulla, which contains $85 \%$ epinephrine. Rarely, tumours produce epinephrine. In some cases the clinical picture is dominated by signs of beta-receptor stimulation, such as tachycardia and hypermetabolism.

Patients present with symptoms which reflects excessive secretion of norepinephrine, epinephrine and dopamine into the circulation. Patients who secrete norepinephrine have hypertension, which is severe and refractory to conventional therapy. Those with epinephrine present with tachycardia, palpitations, panic attacks and feeling of doom. ${ }^{[2]}$ The differences in catecholamine release may contribute to clinical differences in the secretion of neurotransmitters or hormones with subsequent clinical presentation, also tumour size correlates with the ratio of free catecholamine.[7] Patients with small tumours tend to have low concentration of catecholamines with high turnover and low urinary VMA: Catecholamines ratio. Conversely, large tumours tend to have high concentration of catecholamines with high turnover low urinary VMA: Catecholamine ratio. ${ }^{[4]}$

\section{Diagnosis of Pheochromocytoma}

PCC is encountered by the Anaesthetists under Two Circumstances

1. The diagnosed patient scheduled electively for extirpation of PCC.

2. Incidental: Emergent situation in which cardiovascular manifestations of catecholamines excess occur during the course of surgery, which is not related to PCC.

The standard method of confirming the diagnosis is by measuring the urinary catecholamines and their metabolites in a 24-hour collection sample. Total urinary catecholamine excretion exceeding $300 \mu \mathrm{g} /$ day is diagnostic, provided the patient is symptomatic or hypertensive at the time of collection. ${ }^{[4]}$ At present the diagnostic tests of choice is the measurement of fractional plasma and urine metanephrines which are highly sensitive tests (100\%) for sympathetic chromaffin tumour. Methods using mass spectrometry to measure plasma free metanephrines appear to be superior.[3] Fractional plasma free metanephrines are more sensitive than 24-hour urinary metanephrines and catecholamines. ${ }^{[8]}$ Though 24-hour urine metanephrine and catecholamine levels provide clinically acceptable and better sensitivity than fractional plasma free metanephrine values, in paediatrics it is difficult to collect complete 24-hour urine sample, hence fractionated free metanephrines should be considered the biochemical test of choice in this population. ${ }^{[8]}$

\section{Localisation}

Once the diagnosis of PCC has been confirmed by urinary catecholamines, CT and MRI both provide accurate and consistent identification of majority of pheochromocytoma. MIBG ( $\left.{ }^{131}\right)$ metaiodobenzylguanidine has been effective in identifying uncommon sites such as urinary bladder and pericardium, but it has a disadvantage that it may be taken up by neuroblastomas, medullary thyroid carcinomas, carcinoids and small cell carcinomas of lungs.

\section{Preoperative Pharmacological Control}

The aim is to vasodilate and increase the vascular volume in presence of extremely constricted vascular capacity. It is mandatory to control the hypertension, hyperglycaemia, tachycardia, dysrhythmias and optimisation of the cardiovascular function. Once the diagnosis is done, medical therapy should be started 1 to 2 weeks before the surgery to minimise the perioperative complications during induction and manipulation of tumour. The drug of choice is noncompetitive $\alpha$-blocker phenoxybenzamine 0.2 to 1 $\mathrm{mg} / \mathrm{kg} /$ day in divided doses.[3] 
Although, surgical removal is the definitive treatment of PCC, pharmacological therapy plays a critical role in control of BP both perioperatively and those patients with inoperable diseases; $\alpha$ and $\beta$-blockers often in combination are used to control the ventricular tachyarrhythmias and hypertension.

\section{Phenoxybenzamine}

It has been widely used since 1950s with a long duration of action, acts by non-competitive blockade preventing the effects of surges of catecholamine release during preparatory period. Pharmacological half-life is 24 hours, the prolongation of the receptor blockade depends on the rate of resynthesis of receptors. Marked prolongation of $\alpha$-blockade is seen in the post-operative period despite stopping the administration of phenoxybenzamine 24 - 48 hours before the surgery. Side effects are somnolence, extensive peripheral oedema, headache, stuffy nose and postural hypotension.

\section{Prazosin}

Selective competitive $\alpha 1$ adrenoreceptor blocker. It was advocated by Wallace and Gill,[9] but failed to achieve popularity, may be due to inadequate doses, high clearance $(4.5 \mathrm{~mL} / \mathrm{min} / \mathrm{kg})$ and short elimination half-life ( 2 - 3 hours). Thus, reducing the prazosin blood concentration, if the last dose was given the previous night. Russell and colleagues claim that phenoxybenzamine pre-treatment provided superior intraoperative stability than prazosin.[10]

\section{Doxazosin}

Competitive and selective $\alpha 1$ agonist, non-lipophilic, high bioavailability, does not cross blood brain barrier and long duration of action. Thus, one dose per day is sufficient. It does not block the presynaptic $\alpha 2$ adrenoreceptors, which regulate norepinephrine release at cardiac adrenergic nerve endings. There is no need to administer $\beta$-blockers unless the patient has an epinephrine secreting tumour.

The selective $\alpha-1$ antagonists offer several advantages as these drugs do not elicit reflex tachycardia, have shorter duration of action and less postural hypotension.

$\beta$-adrenoreceptor antagonists: To limit the symptoms and signs of increased circulating epinephrine, mainly tachycardia with or without arrhythmias. To block excessive cardiac sympathetic drive secondary to suppression of the presynaptic $\alpha 2$ regulating mechanism. Example: Atenolol, Labetalol, Carvedilol and Propranolol. Suppression of $\beta$ adrenoreceptor mediated cardiac sympathetic activity in the absence of adequate arteriolar dilatation may precipitate acute pulmonary oedema.

Other drugs: Calcium channel blockers- Nifedipine; ACE inhibitors- Ramipril; Angiotensin antagonists- Losartan.

\section{MATERIALS AND METHODS}

The study was designed as a retrospective case series and comparative analysis in a single institute and our institutional experience with challenges during perioperative management of pheochromocytoma. Over a period of time various techniques and drugs have changed the management of pheochromocytoma, yet no single technique or drug has proved to be ideal. Hence, we decided to do this study with an objective of analysing our institutional progress and experience in anaesthetic management of PCC hoping to find any better options that can be helpful in future. We managed
7 children aged between 8 and 14 years with pheochromocytoma over a period of 9 years from 2006 to 2015. Sustained hypertension was very common in children with classic triad of headache, palpitations and diaphoresis. Pallor, orthostatic hypotension, syncope, tremor and anxiety also occurred in few. Four of the seven patients presented with hypertensive encephalopathy, cardiomyopathy, malignant papilloedema and one had features of WolfHirschhorn syndrome. Two patients had bilateral PCC.

Management of anaesthesia for pheochromocytoma and paraganglioma depends on the surgical approach. Traditionally, it has been performed by open lateral retroperitoneal surgery through a transabdominal approach. Recently laparoscopic transperitoneal approach has become popular, which reduces post-operative pain and hospitalisation in the absence of complications resulting from persistent adrenergic blockade.[2]

Goals of our anaesthetic management was to provide optimal surgical conditions and suppress the responses to endotracheal intubation, surgical stimulation, tumour handling and revascularisation. Combined epidural and general anaesthesia was our preferred technique. Thiopentone and propofol were used as induction agents and Atracurium as muscle relaxant. Opioids are preferred due to better haemodynamic profile. Other drugs which we added in our armamentarium are dexmedetomidine and $\mathrm{MgSO}_{4}$.

In our series of 8 cases of pheochromocytoma over ten years following the basic techniques, slowly we have changed to sevoflurane and isoflurane from halothane. Preoperatively, phenoxybenzamine was stopped 48 hours before the surgery. Oral premedication was done with diazepam in all the patients (night $5 \mathrm{mg}$ and morning $5 \mathrm{mg}$ orally). In the operating room after placing in position, non-invasive monitors like ECG, $\mathrm{SPO}_{2}$ probe, NIBP and $\mathrm{EtCO}_{2}$ were attached. Induction was done with IV thiopentone, fentanyl or morphine, ondansetron and atracurium, intubated with cuffed endotracheal tube and maintained on circle absorber circuit. Before intubation, injection Xylocard $1.5 \mathrm{mg} / \mathrm{kg}$ was given to obtund the intubation response.

Radial artery cannulation and internal jugular vein catheterisation were done in all the cases for the monitoring of CVP and arterial BP. Anaesthesia was maintained by oxygen, air, isoflurane, positive pressure ventilation and atracurium infusion of $1.5 \mathrm{mg} / \mathrm{kg} /$ hour. Surgical incision was usually at mid and low thoracic segments. 0.03 $\mathrm{mL} / \mathrm{kg} /$ segment of $0.125 \%-0.25 \%$ bupivacaine was injected into the epidural space as bolus after placing the catheter followed by top-up of bupivacaine hourly. Patients were then turned to the respective side of the operation for the laparoscopic port insertion whenever planned. No changes in the HR and BP during shifting or turning of the patient were recorded in all our cases. For laparoscopic surgery, patient was placed in full lateral position with tumour site on the upper most. Once the epidural block was effective, we noted that further doses of fentanyl or neuromuscular blocking drugs were less used in open surgical techniques, but in laparoscopic surgery the requirement of muscle relaxant was more probably due to the drive to breathe as more $\mathrm{CO}_{2}$ is absorbed from the peritoneal cavity.[2]

A combination of regional anaesthesia with general anaesthesia and adequate opioid dose, is sufficient to prevent the intubation stress response, surgical response to skin 
incision and exposure of tumour. But for tumour manipulation however gently performed by the experienced surgeon causes haemodynamic changes during surgery. In such situation, we used Magnesium Sulphate $15 \mathrm{mg} / \mathrm{kg}$ as bolus dose or Dexmedetomidine alpha agonist infusion was started in same patients at the rate of 1 to $2 \mu \mathrm{g} / \mathrm{kg} / \mathrm{hr}$ and increased up to $3 \mathrm{ug} / \mathrm{kg} /$ hour if needed. Esmolol infusion was given to one patient in order to reduce the HR response, as it is ultrashort acting when no response was seen to dexmed and magnesium sulphate. Immediately after ligation of adrenal vein, BP started falling in unilateral pheochromocytoma cases which was treated effectively with inotropes. We extubated all patients on table uneventfully with paediatric intensive care unit monitoring for next 48 hours.

\section{RESULTS}

We were successful in the perioperative management of all children. Two of the seven patients underwent intraoperative conversion of surgery to open laparotomy, two had severe post-operative hypotension, which was well managed on time and recurrence of pheochromocytoma was seen in one patient. All children were normotensive when discharged. There were no deaths reported on long-term followup.

Supplementary doses of magnesium sulphate was required in all of our patients at the time of tumour manipulation. In 2 patients, magnesium sulphate was ineffective in blocking catecholamine release, which was documented by plasma levels of catecholamines measured during surgery. Magnesium sulphate was used in all seven cases, but in two cases we could not achieve control on hypertension during tumour resection, instead esmolol infusion $150 \mu \mathrm{gram} / \mathrm{kg} /$ hour was used.

In our series of 7 cases of pheochromocytoma- in 2 cases (earlier) dexmedetomidine alpha agonist was not used due to unavailability, in other 5 cases dexmedetomidine infusion of 1 to $2 \mathrm{ug} / \mathrm{kg} /$ hour was started from the beginning of the surgery and given as boluses whenever required during manipulation. In one case, there was no control of BP which was managed with Esmolol infusion.

In our case series, post-operative hypotension was managed with fluid resuscitation; inotropes like dopamine, dobutamine, adrenaline and noradrenaline infusions in different patients as per requirement. In two patients, there was no hypotension.

\section{DISCUSSION}

PCC is a dusky-coloured rare tumour of chromaffin tissue. Chromaffin refers to the brown-black colour resulting from the oxidation of catecholamines after staining with chromium salts. The rigorous preoperative treatment minimises the haemodynamic variations during surgeries. Collaboration between the surgeon and anaesthesiologist is of great importance. The use of drugs that increases sympathetic tone such as ketamine, pancuronium and desflurane must be avoided. Anaesthesia induction and tracheal intubation must be smooth to avoid hypertension and tachycardia. Several drugs and techniques are proposed to blunt responses such as propofol, lignocaine, esmolol etc...11]

Opioids are also haemodynamically safe and do not alter cardiac output and heart rate in a dose dependent manner. Remifentanil infusion $0.5 \mu \mathrm{g} / \mathrm{kg} / \mathrm{min}$ can be used, but causes bradycardia and hypotension.[11] Propofol is another anaesthetic and hypnotic drug with short acting effect. Propofol as an infusion (25 to $75 \mu \mathrm{g} / \mathrm{kg} / \mathrm{min}$ ) with remifentanil decreases haemodynamic response during pheochromocytoma resection. The pharmacological profile of these drugs, make total intravenous anaesthesia a modern and safe anaesthetic choice. Another modern option is dexmedetomidine. Its unique pharmacology provides satisfactory preoperative sedation, control of intraoperative haemodynamic changes and reduces the anaesthetic requirements enhancing post-operative analgesia too. It attenuates the sympathetic response to tracheal intubation and emergence from anaesthesia.[11]

Hull[2] quoted that since the first successful operation by the Swiss surgeon Rocex in 1926, almost every possible anaesthetic technique has been advocated and anaesthetic technique should be based on the sound pharmacological principles. We used combined regional and general anaesthetic techniques using mid-to-low thoracic segmental epidural anaesthesia. Magnesium sulphate and dexmedetomidine- alpha agonists for the control of haemodynamic changes in response to tumour manipulation. Shuttler, however, advocated rational management during surgery based on measurement of catecholamines secretions.[12]

The use of a combined epidural and general anaesthesia is considered to be the most suitable and our preferred technique.[5,13] The total intravenous anaesthesia technique using propofol and opioids such as fentanyl and remifentanil is a good option to the traditional inhalational anaesthetic technique. The regional anaesthetic technique although supresses the response of surgical incision, cannot control the quantum of catecholamines released during the tumour handling. Among the inhalational agents, isoflurane and sevoflurane are the preferable agents as they do not sensitise the myocardium to catecholamines. Though, thiopentone and propofol are the commonly used drugs during induction of anaesthesia, propofol is preferred as it produces vasodilatation and blunts the hypertensive response to laryngoscopy and intubation. Vecuronium should be preferred as a muscle relaxant due to its cardiovascular stability. At the end of surgical procedure, it will be solely the anaesthesiologist's decision whether to extubate the patient on table or later in the intensive care unit. The immediate post-operative course and recovery depends upon many intra-operative factors such as clinical status of the patient, eventful haemodynamic episodes, duration of surgery and anticipated stormy post-operative period. Neuromuscular blockade should be reversed by a combination of neostigmine and glycopyrrolate as the antimuscarinic effects of the latter coincide with the onset of cholinergic effects of the former thus producing minimal tachycardia.[2]

In patients pre-treated with phenoxybenzamine, the hypotension was more.[14] Prys-Roberts et al noted that doxazosin receiving patients had less hypotension during the surgical procedure, which returned to baseline levels as soon as the consciousness returned to near normal level and cerebral arousal stimulated the symptoms of nervous system.[14] They also noted that such patients required less fluid loading in postoperative period when compared to the patients who received phenoxybenzamine. In our institute, we do not routinely use doxazosin preoperatively. For all the 
patients, our endocrinologists routinely use phenoxybenzamine, propranolol and calcium channel blockers preoperatively.

\section{Alternative Anaesthetic Technique and Adrenoreceptor Antagonist Strategies}

Hull in his 1986 review, preferred a balanced anaesthetic scheme of enflurane in nitrous oxide supplemented with an alfentanil infusion and sustained neuromuscular block.[15] Hull used a potent vasodilator to control hypertension and a $\beta$-adrenoceptor antagonist.

In our experience isoflurane was equally effective as an arteriolar dilator with rapid onset of action, while avoiding the undesirable metabolic consequences seen in prolonged SNP infusions in paediatrics.

Calcium channel blockers are also used based on the concept that calcium ion transfer is essential for the release of catecholamines from the chromaffin cells of the adrenal medulla and adrenergic nerve endings.[16,17] A French group advocated the use of calcium channel blockers exclusively in the preoperative preparation of patients and there are a number of case reports in which nifedipine and nicardipine have been used as well.[18,19,20] Hull noted that drugs which release histamine in turn provoke catecholamine release from the chromaffin granules.[15] But we have used drugs like morphine and atracurium safely in tumour resection without any side effects. Morphine was also used safely in study by Hamaji et al.[21]

\section{Role of Magnesium Sulphate}

James and colleagues have championed the infusion of magnesium sulphate as the main alternative therapeutic strategy to adrenergic block during anaesthesia and surgery for pheochromocytoma.[22,23] Magnesium ions inhibit the release of catecholamines from the normal adrenal medulla and adrenergic nerve endings.[24,25] James described a series of 17 patients in whom preoperative control was achieved with either phenoxybenzamine (8 patients) or prazosin (8 patients) with intraoperative control achieved by loading dose of magnesium sulphate ( 40 to $60 \mathrm{mg} / \mathrm{kg} / \mathrm{hr}$ ) followed by an infusion of $1-2 \mathrm{gm} /$ hour.[22] The study had recorded serum magnesium concentrations between $2-4 \mathrm{mmol} /$ litre. Good haemodynamic stability was obtained in the study as the arterial pressures were maintained within $30 \mathrm{mmHg}$, range of the pre-operative level in 12 of the 17 patients, and SNP was required in another 5 patients.

In view of the rarity of this disease, such trials of magnesium sulphate with other drug trials are unlikely to be undertaken and in the final analysis anaesthetists will have to rely on their "idiosyncratic fondness of particular drugs or methods."

\section{Role of Dexmedetomidine}

Its unique pharmacology provides a satisfactory preoperative sedation, control of intraoperative haemodynamic changes, reduces the anaesthetic requirements and enhances postoperative analgesia.[11] It also attenuates the sympathetic response to tracheal intubation and emergence from anaesthesia.[26,27] Kallio et al showed that dexmedetomidine decreases arterial blood pressure and heart rate with also reduction in plasma level of norepinephrine.[28,29]

\section{Postoperative Management}

The main concern of postoperative period is the persistent hypotension which may be refractory requiring intravascular volume replacement and adrenoreceptor agonists. This depends upon multiple factors.[2]

- Excised tumour is active.

- The catecholamine output of the contralateral adrenal gland was effectively suppressed.

- The patient's relevant adrenoreceptors were downregulated.

This occurs due to generalised sympathetic nervous activity, which is considerably diminished during anaesthesia as a result of the lack of "arousal" and its effects in stimulating the hypothalamus. Postoperative blood glucose should also be monitored to detect hypoglycaemia.[30]

In 19 patients series by Prys-Roberts et al,[14] patients pre-treated with doxazosin mean (SD) systolic arterial pressure increased by 15 (8) $\mathrm{mmHg}$ following recovery of consciousness to 118 (14) $\mathrm{mmHg}$ by comparison with an increase of 8 (6) $\mathrm{mmHg}$ to 100 (12) $\mathrm{mmHg}$ in 8 patients who had been pre-treated with phenoxybenzamine. The patients in phenoxybenzamine group received $1,200 \mathrm{~mL}$ of gelofusine, but doxazosin group required only $350 \mathrm{~mL}$ of gelofusine, both based on an attempt to maintain CVP at about $8 \mathrm{mmHg}$.

Immediate post-op hypertension can be due to recovery from anaesthetic drugs or pain. The incidence of hypertension can be as high as $50 \%$ for few days in these patients due to elevated catecholamine levels persisting for 7 to 10 days after resection and sometimes a residual tumour may be responsible for the hypertension. [5]

\section{Comparison between Adult and Paediatric Pheochromocytoma}

Paediatric pheochromocytomas are more associated with syndromes and usually multiple. Diagnosis is not difficult if symptoms are appreciated, especially hypertension. In children hypertension is more severe and sustained than in adults leading to contracted intravascular volume.[31] Cardiac arrhythmias are rare, because they mainly secrete norepinephrine.

In the literature, $70 \%-80 \%$ of childhood PCMs arise in the adrenal medulla and thoracic tumours are rare. The prognosis in children with successfully resected tumour depends on the benign or malignant characteristics, the presence of associated conditions and recurrences.[31,32] Various guidelines have been formed for management of PCC and PGL, $[3,34,35]$ yet it needs an individualised approach. Laparoscopic approach is recently considered beneficial in tumour resection.[36-39] Tumour recurrence can pose a great challenge to both anaesthetist and surgeon even after successful primary resection.[40]

\section{CONCLUSION}

In conclusion, we suggest that a prompt early diagnosis, meticulous planning and preparedness to tackle inevitable drop in pressures after complete excision of tumour with vasopressor is necessary. Multidisciplinary approach is the key essential in reducing the mortality and morbidity.

Despite many advances, there is no specific anaesthetic protocol and consistent recommendations from the literature for pheochromocytoma management. A conscientious 
attentive anaesthesia in a well-prepared patient is more important than which drug or drug combination is selected.

\section{ACKNOWLEDGEMENT}

We would like to thank Dr. PM Chandrasekara for the immense support and assistance.

\section{REFERENCES}

[1] Prabhu M, Joseph TT, Shetty N, et al. Child with bilateral pheochromocytoma and a surgically solitary kidney: anaesthetic challenges. Saudi J Anaesth 2013;7(2):197-9.

[2] Prys-Roberts C. Pheochromocytoma - recent progress in its management. Br J Anaesth 2000;85(1):44-57.

[3] Waguespack SG, Rich T, Grubbs E, et al. A current review of the etiology, diagnosis and treatment of paediatric pheochromocytoma and paraganglioma. J Clin Endocrinol Metab 2010;95(5):2023-37.

[4] Vuguin PM, Coppes MJ. Pediatric pheochromocyoma: background, pathophysiology, etiology. Medscape reference. Article 988. 2015.

[5] Bajwa SS, Bajwa SK. Implications and considerations during pheochromocytoma resection: a challenge to anaesthesiologist. Indian J Endocrinol Metab 2011;15(Suppl 4):S337-44.

[6] Subramaniam R. Pheochromocytoma - current concepts in diagnosis and management. Trends in Anesthesia and Critical Care 2011;1(2):104-10.

[7] Imperato-McGinley J, Gautier T, Ehlers $\mathrm{K}$, et al. Reversibility of catecholamine-induced dilated cardiomyopathy in a child with a pheochromocytoma. N Engl J Med 1987;316(13):793-7.

[8] Kudva YC, Sawka AM, Young WF. Clinical review 164: the laboratory diagnosis of adrenal pheochromocytoma: the Mayo clinic experience. J Clin Endocrinol Metab 2003;88(10):4533-9.

[9] Wallace JM, Gill DP. Prazosin in the diagnosis and treatment of pheochromocytoma. JAMA 1978;240(25):2752-3.

[10] Russell WJ, Metcalfe IR, Tonkin AL, et al. The preoperative management of pheochromocytoma. Anaesth Intens Care 1998;26(2):196-200.

[11] Domi R, Laho H. Management of pheochromocytoma: Old ideas and new drugs. Niger J Clin Pract 2012;15(3):253-7.

[12] Shüttler J, Westhofen P, Kania U, et al. Quantitative assessment of catecholamine secretion as a rational principle of Anaesthesia Management in pheochromocytoma surgery. Anaesth Intns Notfall Schmerz 1995;30(6):341-9.

[13] Roizen MF, Horrigan RW, Koike M, et al. A prospective randomized trial of four anaesthetic technique for resection of pheochromocytoma. Anaesthesiology 1982;57:A43.

[14] Prys-Roberts C, Farndon JR. Efficacy and safety of Doxazosin for perioperative management of patients with pheochromocytoma. World Journal of Surgery 2002;26(8):1037-42.

[15] Hull CJ. Pheocromocytoma: diagnosis, preoperative preparation and anesthetic management. Br J Anaesth 1986;58(12):1453-68.
[16] Von Euler US, Lishajko F. Effects of $\mathrm{Mg} 2+$ and $\mathrm{Ca} 2+$ on Noradrenaline release and Uptake in adrenergic nerve granules in different media. Acta Physiol Scand 1973;89(3):415-22.

[17] Douglas WW, Rubin RP. The mechanism of catecholamine release from the adrenal medulla and the role of Calcium in stimulus-secretion coupling. J Physiol 1963;167(2):288-310.

[18] Proye C, Thevenin D, Cecat P, et al. Exclusive use of calcium channel blockers in preoperative and intraoperative control of pheochromocytoma: haemodynamics and free catocholamine assays in ten consecutive patients. Surgery 1989;106(6):1149-54.

[19] Arai T, Hatono $\mathrm{Y}$, Ishida $\mathrm{H}$, et al. Use of nicardipine in the anaesthetic management of pheochromocytoma. Anaesth Analg 1986;65(6):706-8.

[20] Mugawar M, Rajender Y, Purohit AK, et al. Anaesthetic management of Von Hippel-Lindau Syndrome for exscision of cerebellar haemangioblastoma and pheochromocytoma surgery. Anaesth Analg 1998;86(3):673-4.

[21] Hamaji M, Oka N, Tashiro C, et al. Anaestheic management with morphine in pheochromocytoma. Can Anaesth Soc J 1984;31(6):681-6.

[22] James MF. Use of magnesium sulphate in the anaesthetic management of phaeochromocytoma: a review of 17 anaesthetics. Br J Anaesth 1989;62(6): 616-23.

[23] James MFM, Huddle KRL, Owen AD, et al. The use of $\mathrm{MgSO}_{4}$ in the anaesthetic management of phechromocytoma in Pregnancy. Can J Anaesth 1988;35:278-82.

[24] Dougas W, Rubin RP. The mechanism of catecholamine release from the adrenal medulla and the role of calcium in stimulus secretion coupling. J Physiol 1963;167(2):288-310.

[25] Lishajko F. Releasing effect of calcium and phosphate on catecholamines, ATP and protein from chromaffin cell granules. Acta Physiol Scand 1970;79(4):575-84.

[26] Mukhtar AM, Obayah EM, Hassona AM. The use of dexmeditomedine in pediatric cardiac surgery. Anaesth Analg 2006;103(1):52-6.

[27] Talke P, Chem R, Thomas B, et al. The haemodynamic and adrenergic effects of perioperative dexmeditimedine infusion after vascular surgery. Anaesth Analg 2000;90(4):834-9.

[28] Khetarpal M, Yadav M, Kulkarni D, et al. Role of dexmedetomidine and sevoflurane in the intraoperative management of patient undergoing resection of phaeochromocytoma. Indian J Anaesth 2014;58(4):496-7.

[29] Kallio A. Scheinin M, Koulu M, et al. Effects of dexmeditomedine a selective alpha 2 adrenoreceptor agonist, on haemodynamic control mechanisms. Clin Pharmacol Ther 1989;46(1):33-42.

[30] Martin R, St-Pierre B, Molliner OR. Phaeochromocytoma and postoperative hypoglycaemia. Canad Anaesth Soc J 1979;26(4):2602. 
[31] Ein SH, Pullerits J, Creighton R, et al. Pediatric pheochromocytoma. A 36-year review. Pediatric Surg Int 1997;12(8):595-8.

[32] Ramakrishna H. Pheochromocytoma resection: current concepts in anesthetic management. Journal of Anaesth Clin Pharmc 2015;31(3):317-23.

[33] Lenders JW, Duh QY, Eisenhofer G, et al. Pheochromocytoma and paraganglioma: an endocrine society clinical practice guideline. J Clin Endocrinol Metab 2014;99(6):1915-42.

[34] Mazza A, Armigliato M, Marzola MC, et al. Antihypertensive treatment in pheochromocytoma and paraganglioma: current management and therapeutic features. Endocrine 2014;45(3):469-78.

[35] Mannelli M. Management and treatment of pheochromocytomas and paragangliomas. Ann N Y Acad Sci 2006;1073:405-16.
[36] Conzo G, Musella M, Corcione F, et al. Laparoscopic adrenalectomy, a safe procedure for pheochromocytoma. A retrospective review of clinical series. Int J Surg 2013;11(2):152-6.

[37] Kulis T, Knezevic N, Pekez M, et al. Laparoscopic adrenalectomy: lessons learned from 306 cases. J Laparoendosc Adv Surg Tech A 2012;22(1):22-6.

[38] Nguyen PH, Keller JE, Novitsky YW, et al. Laparoscopic approach to adrenalectomy: review of perioperative outcomes in a single center. The Am Surg 2011;77(5):592-6.

[39] Gumbs AA, Gagner M. Laparoscopic adrenalectomy. Best Pract Res Clin Endocrinol Metab 2006;20(3):48399.

[40] Plouin PF, Chatellier G, Fofol I, et al. Tumor recurrence and hypertension persistence after successful pheochromocytoma operation. Hypertension 1997;29(5):1133-9. 